\title{
Random allogeneic blood transfusion in pigs: characterisation of a novel experimental model
}

\author{
Alexander Ziebart ${ }^{\text {Corresp., } 1}{ }^{1}$, Moritz Schaefer ${ }^{1}$, Rainer Thomas ${ }^{1}$, Jens Kamuf ${ }^{1}$, Andreas Garcia-Bardon ${ }^{1}$, Christian \\ Moellmann $^{1}$, Robert Ruemmler ${ }^{1}$, Florian Heid ${ }^{1}$, Arno Schad ${ }^{2}$, Erik K Hartmann ${ }^{1}$ \\ ${ }^{1}$ Department of Anesthesiology, Medical Centre of the Johannes Gutenberg-University, Mainz, Germany \\ 2 Institute of Pathology, Medical Centre of the Johannes Gutenberg-University, Mainz, Germany \\ Corresponding Author: Alexander Ziebart \\ Email address: alexander.ziebart@unimedizin-mainz.de
}

\begin{abstract}
:
Background: Organ cross-talk describes interactions between a primary affected organ and a secondarily injured remote organ, particularly in lung-brain interactions. A common theory is the systemic distribution of inflammatory mediators that are released by the affected organ and transferred through the bloodstream. The present study characterises the baseline immunogenic effects of a novel experimental model of random allogeneic blood transfusion in pigs designed to analyse the role of the bloodstream in organ cross-talk.
\end{abstract}

Methods: After approval of the State and Institutional Animal Care Committee 20 anesthetized pig were randomized in a donor and an acceptor (each $n=8$ ): the acceptor animals each received high-volume whole blood transfusion from the donor (35-40 ml kg-1). Four animals received balanced electrolyte solution instead of blood transfusion (control group; $n=4$ ). Afterwards the animals underwent extended cardiorespiratory monitoring for eight hours. Post mortem assessment included pulmonary, cerebral and systemic mediators of early inflammatory response (IL-6, TNF-alpha, iNOS), wet to dry ratio, and lung histology.

Results: No adverse events or incompatibilities occurred during the blood transfusion procedures. No hints for systemic inflammatory response were found and the pulmonary function was not affected through blood transfusion. Lung histopathology scoring did not display relevant intergroup differences. Neither within the lung nor within the brain an up-regulation of inflammatory mediators was detected. High volume random allogeneic blood transfusion in pigs neither impaired pulmonary integrity nor induced systemic, lung, or brain inflammatory response.

Conclusion: This approach can represent a novel experimental model to characterize the blood-bound transmission in remote organ injury. 


\section{experimental model}

3

4 Alexander Ziebart ${ }^{*}$, Moritz Schaefer ${ }^{1}$, Rainer Thomas ${ }^{1}$, Jens Kamuf ${ }^{1}$, Andreas Garcia-Bardon ${ }^{1}$, $5 \quad$ Christian Moellmann, Robert Ruemmler ${ }^{1}$, Florian Heid ${ }^{1}$, Arno Schad $^{2}$, Erik K. Hartmann ${ }^{1}$

$7{ }^{1}$ Department of Anesthesiology, Medical Centre of the Johannes Gutenberg-University, Mainz, 8 Germany

92 Institute of Pathology, Medical Centre of the Johannes Gutenberg-University, Mainz, Germany

*Corresponding author:

12 alexander.ziebart@unimedizin-mainz.de

13 
25

26

27

28

29

30

\section{Abstract:}

Background: Organ cross-talk describes interactions between a primary affected organ and a secondarily injured remote organ, particularly in lung-brain interactions. A common theory is the systemic distribution of inflammatory mediators that are released by the affected organ and transferred through the bloodstream. The present study characterises the baseline immunogenic effects of a novel experimental model of random allogeneic blood transfusion in pigs designed to analyse the role of the bloodstream in organ cross-talk.

Methods: After approval of the State and Institutional Animal Care Committee 20 anesthetized pigs were randomized in a donor and an acceptor group (each $n=8$ ): the acceptor animals each received high-volume whole blood transfusion from the donors $\left(35-40 \mathrm{ml} \mathrm{kg}^{-1}\right)$. Four animals received balanced electrolyte solution instead of blood transfusion (control group; $\mathrm{n}=4$ ). Afterwards the animals underwent extended cardiorespiratory monitoring for eight hours. Post mortem assessment included pulmonary, cerebral and systemic mediators of early inflammatory response (IL-6, TNF-alpha, iNOS), wet to dry ratio, and lung histology. Results: No adverse events or incompatibilities occurred during the blood transfusion procedures. Systemic cytokine levels and pulmonary function were unaffected. Lung histopathology scoring did not display relevant intergroup differences. Neither within the lung nor within the brain an up-regulation of inflammatory mediators was detected. High volume random allogeneic blood transfusion in pigs neither impaired pulmonary integrity nor induced systemic, lung, or brain inflammatory response.

Conclusion: This approach can represent a novel experimental model to characterize the bloodbound transmission in remote organ injury. 


\section{Introduction:}

50 Single organ injury or failure does not exert an isolated effect on the primary affected organ system. Remote organs consecutively develop secondary injuries that are not connected to the

initial insult. This is referred to as deleterious organ cross-talk and described particularly for lung and brain interactions, where this pathomechanism is well known, but not fully understood. E.g. mechanical ventilation in the intensive care unit may cause inflammatory response and induce damage to distant organs. On the other hand, severe brain injury is associated with an increased risk of lung failure (Mascia et al. 2007; Pelosi \& Rocco 2011).

Different theories for this phenomenon consider neuronal response, hormone release or circulating inflammatory mediators as causative (Han 2017; Mrozek et al. 2015; Pelosi \& Rocco 2011). The hypothesis of inflammatory mediator distribution is promising as several studies show inflammatory response after a primary hit. The blood stream seems to be the distributor in remote organ injury and cross-talk (Hegeman et al. 2009; Klein et al. 2016; Mrozek et al. 2015). In theory, inflammatory cytokines that are released into the systemic circulation induce remote organ injury. Hence, for experimental purposes this effect might be transferable from one individual to another via whole blood transfusion. Porcine models, in this context, offer advantages in experimental assessment of blood stream-related distribution of inflammatory mediator transmission. Firstly, higher blood volumes can be transferred in comparison to common laboratory rodent species. Secondly, because major inter-individual blood incompatibilities are missing (Smith et al. 2006). Only few reports reveal bronchospasm, disseminated intravascular coagulation, bleeding, or progressive hypotension during blood transfusion in pigs (Hunfeld et al. 1984; Sheil et al. 1972; 
70 Smith et al. 2006). This porcine system is based on blood groups A0, whereas the human group B

71 is missing. Additional 16 different antigens are known to form the subgroups A-P (Smith et al.

72 2006). Unlike the human Rhesus antigen, which is highly immunogenic and prone for transfusion

73 reactions or hemolysis, the porcine Rhesus antigen is coded by a single gen without any

74 polymorphisms and less pathologic relevance (Avent \& Reid 2000; Omi et al. 2003). However,

75 the distinct effects of porcine blood transfusion with focus on end-organ inflammatory response

76 are barely examined. In order to further elucidate the role of the blood stream as distributor in

77 remote organ injury the present study aims to characterize the baseline immunogenic effects of

78 random allogeneic blood transfusion in pigs. The approach may allow for experimental separation

79 of blood-bound effects on organ integrity from an already injured subject: hence, we hypothesized

80 that single, large-volume blood transfusion from a healthy donor neither exhibits inflammatory

81 response nor impairs the pulmonary function in the acceptor. As secondary hypotheses, the

82 systemic and cerebral inflammatory response is analyzed.

84 Materials \& Methods

85 The prospective-randomized animal study was approved by the State and Institutional Animal

86 Care Committee (Landesuntersuchungsamt Rheinland-Pfalz, Koblenz, Germany; approval

87 number: 23 177-07/G 14-1-084). The study was conducted in accordance with the ARRIVE

88 guidelines and the international guidelines for the care and use of laboratory animals. The

89 experiments were performed from 12/2014 till 3/2015. Twenty juvenile German Landrace pigs

90 (sus scrofa domestica, weight $28 \pm 2 \mathrm{~kg}$ ) were examined. Eight animals served as blood donors.

91 Eight animals received allogenic blood transfusion. The control group consisted of four animals

92 that received the same amount of balanced electrolyte solution instead of blood transfusion. One 
93 acceptor animal was excluded due to complicated airway management and multiple intubation

94 attempts with subsequent respiratory failure before the transfusion. Per protocol and approval, the

95 donor animals that inevitably suffered from hemorrhagic shock were subsequently included in

96 another study protocol focusing on fluid resuscitation. All experiments were conducted under

97 continuous general anesthesia. This manuscript adheres to the applicable EQUATOR guidelines.

98

Anesthesia and instrumentation

Anesthesia and instrumentation procedure was previously described in detail in our former studies

(Ziebart et al. 2015; Ziebart et al. 2018): Briefly, in each case two animals received an intramuscular injection of ketamine $\left(8 \mathrm{mg} \mathrm{kg}^{1}\right)$ and midazolam $\left(0.2 \mathrm{mg} \mathrm{kg}^{-1}\right)$ followed by induction of general anesthesia via an ear vein cannula (propofol $4 \mathrm{mg} \mathrm{kg}^{-1}$, and fentanyl $4 \mu \mathrm{g} \mathrm{kg}^{-1}$, atracurium $0.5 \mathrm{mg} \mathrm{kg}^{-1}$ ), which was maintained by continuous infusion (propofol $8-12 \mathrm{mg} \mathrm{kg}^{-1} \mathrm{~h}^{-}$ , fentanyl 0.1-0.2 $\mathrm{mg} \mathrm{h}^{-1}$ ). After orotracheal intubation the animals were ventilated in volumecontrolled mode (VCV; AVEA, CareFusion, USA): positive end-expiratory pressure (PEEP) 5 $\mathrm{cmH}_{2} \mathrm{O}$, tidal volume $7 \mathrm{ml} \mathrm{kg}^{-1}$, inspiration to expiration ratio $1: 2$, fraction of inspired oxygen $\left(\mathrm{FiO}_{2}\right) 0.4$ and variable respiration rate to achieve an end-tidal $\mathrm{CO}_{2}<45 \mathrm{mmHg}$. In case of respiratory insufficiency, mechanical ventilation parameters escalated according to an adaption of the ARDS network tables. (Ziebart et al. 2014) Afterwards, the animals were randomized into an acceptor and a donor animal. Ultrasound guided femoral vascular access under sterile conditions

112 included a central venous line, a pulse contour cardiac output catheter (PiCCO, Pulsion Medical, 113 Germany), and a pulmonary arterial catheter in the acceptor animals. The donor animals received 114 a central venous catheter, an arterial line, a pulse contour cardiac output system (PiCCO, Pulsion 115 Medical Systems, Germany) and a large-bore venous introducer. Extended hemodynamics, 
116 transpulmonary thermodilution-derived parameters, spirometry, and gas exchange were 117 continuously monitored (Datex S/5 Monitor, GE Healthcare, Germany; PiCCO, Pulsion Medical

118 Systems, Germany). Hematological and blood gas measurements were repetitively conducted.

119 Electrical impedance tomography (EIT; Goe-MF II, CareFusion, Germany) was used to measure 120 variations of the thoracic bioimpedance that are associated with pulmonary aeration. Sixteen 121 adhesive electrodes were placed around the thorax and the ventilation distribution was measured 122 for three lung compartments (Level 1-3; non-dependent, central, dependent) (Bodenstein et al. 123 2012; Ziebart et al. 2014). Multiple breath-nitrogen washout/washin technique was applied to 124 measure the functional residual capacity (Kamuf et al. 2017). Body temperature was measured by 125 a rectal probe and a surface warming device prevented hypothermia. All animals received a 126 background infusion of $5 \mathrm{ml} \mathrm{kg}^{-1} \mathrm{~h}^{-1}$ balanced electrolyte solution (Sterofundin, B.Braun, 127 Germany).

128 After anesthesia, instrumentation, and 20-minute recovery period baseline values were measured 129 and recorded.

\section{Blood withdrawal procedure}

132 From the donor animals $35-40 \mathrm{ml} \mathrm{kg}^{-1}$ blood was taken for allogenic blood transfusion through the 133 arterial catheter. The whole blood was collected in specific collector bags system (Composelect, 134 Fresenius-Kabi AG, Homburg Germany). These bags comprised $63 \mathrm{ml} \mathrm{CPD/100} \mathrm{ml} \mathrm{SAG-M} \mathrm{-}$ 135 RCC (Citrate phosphate dextrose, Erythrocyte storage in hypertonic conservation medium). After 136 collection in a primary bag. The blood was drained through a leucocyte-depleting filter into a 137 second bag that contains the anticoagulants. Finally, the whole blood was collected into a bag with 138 a connector for a transfusion system. Afterwards the whole blood was stored by room temperature 
139 for about ten minutes before transfusion to avoid negative effects of long-time storing or cooling.

140 Afterwards the donor animals were transferred to the fluid resuscitation protocol that was included

141 in the same approval: they were euthanized by intravenous injection of propofol (200mg) and

142 potassium chloride (40mval) within the scope of this protocol. (Ziebart et al. 2018)

144 Experimental protocol:

145 The experimental protocol is summarized in figure 1. Immediately after blood withdrawal the

146 whole blood was transfused into the recipients through the venous vascular access sheath over 15

147 minutes through a transfusion system with an integrated aggregate-filter. A control group ( $n=4)$

148 underwent the identical instrumentation and protocol, though received an equal amount of

149 balanced electrolyte solution instead of allogeneic blood transfusion. Afterwards the animals were

150 monitored for eight hours. In case of hemodynamic deterioration (mean arterial pressure $<60$

$151 \mathrm{mmHg}$ ) continuous noradrenaline infusion was administered. After finishing the protocol, the

152 animals were killed in deep general anesthesia by injection of propofol (200 mg) and potassium

153 chloride (40 mval).

154

155

Post mortem analysis

156 Repetitively taken serum blood samples were used to determine the serum levels of TNF-alpha 157 and IL-6 by enzyme-linked immunosorbent assays (Porcine Quantikine ELISA Kits, R/D Systems, 158 Germany). After finishing the protocol, the lung and the brain each were removed en-bloc. Four 159 representative tissue samples (dependent periphery, dependent central, non-dependent central, 160 non-dependent periphery) from the right lung were extracted, formalin-fixed, and used for 161 histological evaluation of alveolar damage in the upper and the lower lung. After paraffin 
162 embedding and hematoxylin/eosin staining the samples were analyzed in investigator-blinded

163 manner by means of standardized scoring scheme (Ziebart et al. 2015; Ziebart et al. 2014).

164 Additional lung samples were cryopreserved for mRNA expression analysis of inflammatory

165 mediators (IL-6, TNF-alpha, iNOS) using real-time polymerase chain reaction (rt-PCR;

166 Lightcycler 480 PCR System, Roche Applied Science, Germany) as described in detail by

167 Hartmann et al. (Hartmann et al. 2015). The mRNA expression was normalized to peptidylprolyl

168 isomerase A (PPIA). The same technique and markers were used to quantify the cerebral mRNA

169 expression from the right hippocampus and the right frontal cortex. Furthermore, the lung and

170 brain water content were determined through the tissue wet to dry ratio. For this procedure the

171 weight of the removed organs was determined directly and after two days of complete drying.

172

173

174

175

176

177

178

179

180

181

182

183

184

185

\section{Statistics}

All parameters are presented mean and standard deviation $( \pm \mathrm{SD})$ or displayed as plots. The analysis focusses on the relevant time points baseline, $0 \mathrm{~h}, 4 \mathrm{~h} 8 \mathrm{~h}$. Additional data are added as supplemental files. Group effects over time (Transfusion, Control) were compared by two-way analysis of variance (ANOVA) with post-hoc Student-Newman-Keuls-Test. Mann-Whitney-UTest was used to analyze the post mortem parameters. A p-value lower than 0.05 was accepted as significant. The software package SigmaPlot 12.5 (Systat Software, USA) was used.

\section{$\underline{\text { Results }}$}

The estimated total blood volume of the pigs $\left(74 \mathrm{ml} \mathrm{kg}^{-1}\right.$ ) was $2084 \pm 166 \mathrm{ml}$ (Lyhs \& Wachtel 1966). The transfused blood volume was $48 \pm 4 \%$. No adverse events or major incompatibilities 
186 occurred during the blood transfusion procedures. Beneath a temporary increase of the mean

187 pulmonary arterial pressure following blood transfusion and a slight increase of the central venous

188 pressure the cardiopulmonary monitoring and blood gas analyses showed no relevant differences

189 between the transfusion and control animals over eight hours (table 1, 2 \& 3). The lactate level

190 was in a normal range and even decreased within eight hours. Through blood transfusion

191 hemoglobin, hematocrit and erythrocyte counts significantly increased without relevant changes

192 in leucocyte amounts (figure 2). Marker of systemic inflammatory response did not differ between

193 the groups as well (figure $2 \& 3$ ). The pulmonary function as measured by blood gas analysis and

194 ventilation distribution was not affected by blood transfusion (table $3 \&$ figure 4). Only the

195 alveolar-arterial oxygen difference increased. At baseline two animal started at a $\mathrm{PaO}_{2} / \mathrm{FiO}_{2}<400$

$196 \mathrm{mmHg}$ without signs of local or systemic illness. A modification of the initial respiratory setting

197 due to hypoxemia was not required in any animal. Lung histopathology scoring did not display

198 relevant intergroup differences (control vs. transfusion; $10 \pm 1$ vs. $11 \pm 3$; each $p>0.05$ ). The tissue

199 wet to dry ratio as surrogate of edema formation between both groups was not increased following

200 transfusion (control vs. transfusion; lung: $4.7 \pm 0.2$ vs. $4.6 \pm 0.2$; brain: $3.3 \pm 0.3$ vs. $3.4 \pm 0.3$; each $p>$

201 0.05). Neither within the lung nor within the brain the mRNA expression analysis revealed an up-

202 regulation of inflammatory markers (figure $5 \& 6$ ).

203

\section{Discussion}

205 The present study establishes a novel model for characterization of remote organ injury related to 206 the distribution of inflammatory mediators through the systemic circulation. We found that one-

207 time high-volume allogeneic blood transfusion in healthy pigs neither impairs pulmonary integrity

208 nor mediates inflammatory response within the lung, brain, or systemic circulation. Accordingly,

209 random allogeneic blood transfusion in pigs is feasible, and per itself does not trigger inflammatory 
210 response in the acceptor animals. Hence, this model could be an appropriate approach to identify

211 the main mediators in injurious organ interactions, i.e. by transfusion of blood from severely brain

212 or lung injured subjects to healthy ones without co-founders. Other experimental protocols with

213 comparable porcine transfusion models investigate questions like acute kidney injury or effects of

214 blood storing and cooling. Several similar but, in some points, different protocols have been

215 described that make use of autologous instead homologous blood, heavier animals to minimize the

216 need for donor animals, or previously induce a hemorrhagic shock to reduce the rate of circulatory

217 overload (Biagini et al. 2017; Masuda et al. 2018; Patel et al. 2011; Wozniak et al. 2018).

218 In principle, blood transfusion can be associated with severe side effects. Beneath

219 incompatibilities, particularly pulmonary complications like transfusion-related acute lung injury

220 (TRALI) or transfusion-associated circulatory overload (TACO) are described. Other systemic

221 side effects include hypotension, anaphylaxis, hemolytic reactions and transfusion-transmitted

222 bacterial infections (Bux \& Sachs 2008). Our study reveals a significant increase of mean arterial,

223 pulmonary arterial and central venous pressure immediately following transfusion, which was

224 underlined by increase of hemoglobin and hematocrit. These symptoms comply with subclinical

225 TACO induced by supraphysiological increase of intravascular volume (Roubinian et al. 2017).

226 Transfusion of longer stored but not freshly drawn blood was shown to considerably increase

227 pulmonary arterial pressure blood, which is presumably an effect of mediators released through

228 storage. (Baron et al. 2012; Solomon et al. 2013) In our study, similar but short-termed effects

229 occurred despite blood transfusion immediately after withdrawal. Furthermore, fluid overload,

230 which inevitably also occurs in our study, is also associated with pulmonary arterial hypertension.

231 (Yilmaz et al. 2016) (Wolsk et al. 2019) As we found no coincident pathologies that allow 
232 distinguishing between fluid overload and mediator-based effects, identification the exact

233 mechanism is beyond the scope of this study.

234 Relevant impairment of the pulmonary gas exchange or alterations in extravascular lung water

235 content, functional residual capacity or ventilation distribution were not observed after blood

236 transfusion. Over eight hours $\mathrm{PaO}_{2} / \mathrm{FiO}_{2}$ decreases to a certain degree going along with a raised

237 alveolar-arterial oxygen difference. In the absence of histopathologic lung injury and during low-

238 grade invasive mechanical ventilation, this is most likely a consequence of atelectasis formation

239 that inevitably occurs during general anesthesia, muscular paralysis and mechanical

240 ventilation.(Hedenstierna \& Edmark 2015) Two animals had a slightly reduced $\mathrm{PaO}_{2} / \mathrm{FiO}_{2}$ ratio $(<$

$241400 \mathrm{mmHg}$ ) already during baseline measurements without any signs for pulmonary or systemic

242 illness: these two animals did neither respond differently to the transfusion nor showed signs of

243 increased histopathologic lung damage despite formally exhibiting a $\mathrm{PaO}_{2} / \mathrm{FiO}_{2}<300 \mathrm{mmHg}$ after

244 eight hours. Increase and withdrawal of intravascular fluid volume in pigs was shown to alter the

245 endexpiratory lung volume as measured by EIT, but did not affect tidal volume or ventilation

246 distribution, which is in line with prior results (Bodenstein et al. 2012). TRALI represents another

247 facet that directly affects the lung following blood transfusion. It is characterized by respiratory

248 distress, hypoxemia, and non-cardiogenic lung edema that is induced by leukocyte-antibodies and

249 neutrophil-priming substances (Bux \& Sachs 2008). These interact with neutrophil granulocytes,

250 which thereby are activated. The resulting clots impair pulmonary capillary perfusion and induce

251 capillary leakage by releasing toxic enzymes and reactive oxygen species (Sachs et al. 2006; Toy

252 et al. 2005). We did not find any TRALI surrogates such as edema formation or increasing counts

253 of leucocytes or specifically neutrophil granulocytes in our porcine model. Furthermore, the

254 histopathological analysis did not reveal relevant inflammatory cell immigration. Additionally, the 
255 regional tidal volume distribution was unaltered, which is described as sign of severe pulmonary

256 illnesses (Chiumello et al. 2013). Gender-specific forms of TRALI are particularly relevant in

257 multiparous women as blood donors (Palfi et al. 2001) (Schmickl et al. 2015). Hence, the exclusive

258 use of male pigs may represent an approach to minimize the TRALI risk. TACO on the other hand

259 tends to appear primarily in patients suffering from serious pre-existing illnesses. In our study

260 healthy animals with intact pulmonary vascular barrier and competent kidney function were used,

261 which enables rapid compensation of the intravascular overload (Bux \& Sachs 2008; Roubinian et

262 al. 2017).

263 Single organ injury or failure can subsequently affect the integrity of distant remote organs leading

264 to multiple organ failure. In particular, deleterious interactions of the lung and the brain have been

265 described (Pelosi \& Rocco 2011). Several models suggest that the lung may play a pivotal role as

266 causative but also remotely injured organ: in mechanically ventilated rats that underwent an

267 aggressive ventilator regime showed remarkable cerebral inflammatory response (Quilez et al.

268 2011). Cyclic variations of the $\mathrm{PaO}_{2}$ caused by non-protective ventilation in lung injured pigs are

269 transmitted to the cerebral tissue and may trigger cerebral injury (Klein et al. 2013; Klein et al.

270 2016). Furthermore, also a cerebral insult is capable to induce or aggravate lung injury (Heuer et

271 al. 2011). Even mechanical ventilation by itself can represent a trigger for cerebral cytokine

272 expression. (Kamuf et al. 2018) Accordingly, we also assessed brain inflammatory response

273 measured by cortical and hippocampal mRNA expression of key inflammatory mediators, which

274 also were unaffected by the transfusion procedure. In comparison to human blood the use of 275 porcine model may offer several advantages for studies concerning remote organ injury: Unlike

276 human blood, where transfusion of incompatible blood products is deleterious, pig blood lacks the

277 human blood group B, the secondary absorption of antigens from the plasma to the blood cells and 
278 the $\mathrm{Rh}$ polymorphism (Omi et al. 2003). Additionally human blood is polygene coded, which

279 results in a much higher incompatibility potential (Smith et al. 2006).

280 The study has several limitations. The study was not designed to assess long-term effects of blood

281 transfusion but to exclude acute side effects of allogeneic blood transfusion itself with regard to a

282 novel experimental approach to elucidate pathomechanisms of deleterious remote organ injury.

283 Logistically it was not possible to investigate different pig subspecies or breeds. Hence,

284 generalization of the reported findings lies beyond the scope of this study. Animal care reasons

285 and the need for additional blood donor animal required a strict limitation of the animal numbers,

286 whereas the chosen group numbers were in line with previous studies inflammatory acute effects

287 in pigs (Hartmann et al. 2015; Ziebart et al. 2014). Exchange of the transfused blood by

288 simultaneous blood withdrawal may be a possibility to avoid fluid overloading. However, given

289 the risk of unpredictable removal of the donor blood, hemodynamic variations, and potential

290 microcirculatory or inflammatory impairment during this complex procedure, we decided to

291 decline this option. Small volume withdrawal prior to the transfusion procedure may represent a

292 reasonable compromise, and may reduce the risk of TACO (Masuda et al. 2018). The use of plasma

293 instead of whole blood is an interesting alternative approach to optimize this setting: theoretically,

294 plasma administration will not increase the hemoglobin content, whereas full concentration of

295 potentially inflammatory mediators should be present. This approach, however, was not feasible

296 for the present study. Additionally, we did not measure cytokine contents in the transfused blood,

297 as the donor animals were healthy and the blood was transfused within minutes. These

298 measurements need to be included in studies focusing on severely injured donor animals.

299

300 Conclusion 
301 In conclusion, high volume random allogeneic blood transfusion in pigs neither impaired

302 pulmonary integrity nor induced systemic, lung, or brain inflammatory response. No further side

303 effects beyond expectable and temporary alterations of hemodynamics and blood count related to

304 the rapid increase of blood volume were detected. Random allogeneic blood transfusion in pigs

305 may represent an experimental model to characterize the blood-bound transmission in remote

306 organ injury.

307

308

309

Acknowledgements:

The authors thank Dagmar Dirvonskis and Dana Pieter for excellent technical support. Parts of

310

311

312

313

314

315

316

317

318

319

320

321

322

323

324

325

326

327

328

329

330

331

332

this study are included in the doctoral thesis of Moritz Schaefer, and content of the professorial

dissertation (habilitation) of Alexander Ziebart. Preliminary results of the study were presented in poster sessions at the German Anesthesia Congress (DAC), Nuernberg, Germany in 2017.

\section{$\underline{\text { References }}$}

Avent ND, and Reid ME. 2000. The Rh blood group system: a review. Blood 95:375-387.

Baron DM, Yu B, Lei C, Bagchi A, Beloiartsev A, Stowell CP, Steinbicker AU, Malhotra R, Bloch KD, and Zapol WM. 2012. Pulmonary hypertension in lambs transfused with stored blood is prevented by breathing nitric oxide. Anesthesiology 116:637-647. 10.1097/ALN.0b013e318246ef77

Biagini S, Dale CS, Real JM, Moreira ES, Carvalho CRR, Schettino GPP, Wendel S, and Azevedo LCP. 2017. Short-term effects of stored homologous red blood cell transfusion on cardiorespiratory function and inflammation: an experimental study in a hypovolemia model. Brazilian Journal of Medical and Biological Research 51:e6258. 10.1590/1414-431x20176258

Bodenstein M, Wang H, Boehme S, Vogt A, Kwiecien R, David M, and Markstaller K. 2012. Influence of crystalloid and colloid fluid infusion and blood withdrawal on pulmonary bioimpedance in an animal model of mechanical ventilation. Physiol Meas 33:1225-1236. 10.1088/0967$3334 / 33 / 7 / 1225$

Bux J, and Sachs UJ. 2008. Pulmonary transfusion reactions. Transfus Med Hemother 35:337-345. 10.1159/000151349

Chiumello D, Froio S, Bouhemad B, Camporota L, and Coppola S. 2013. Clinical review: Lung imaging in acute respiratory distress syndrome patients--an update. Crit Care 17:243. 10.1186/cc13114

Han DW. 2017. Brain and lung: dangerous crosstalk. Korean J Anesthesiol 70:116-117. 10.4097/kjae.2017.70.2.116 
Hartmann EK, Ziebart A, Thomas R, Liu T, Schad A, Tews M, Moosmann B, Kamuf J, Duenges B, Thal SC, and David M. 2015. Inhalation therapy with the synthetic TIP-like peptide AP318 attenuates pulmonary inflammation in a porcine sepsis model. BMC Pulm Med 15:7. 10.1186/s12890-0150002-6

Hedenstierna G, and Edmark L. 2015. Effects of anesthesia on the respiratory system. Best Pract Res Clin Anaesthesiol 29:273-284. 10.1016/j.bpa.2015.08.008

Hegeman MA, Hennus MP, Heijnen CJ, Specht PA, Lachmann B, Jansen NJ, van Vught AJ, and Cobelens PM. 2009. Ventilator-induced endothelial activation and inflammation in the lung and distal organs. Crit Care 13:R182. 10.1186/cc8168

Heuer JF, Pelosi P, Hermann P, Perske C, Crozier TA, Bruck W, and Quintel M. 2011. Acute effects of intracranial hypertension and ARDS on pulmonary and neuronal damage: a randomized experimental study in pigs. Intensive Care Med 37:1182-1191. 10.1007/s00134-011-2232-2

Hunfeld MA, Hoitsma HF, Meijer S, van Haeringen H, and Rietveld FW. 1984. The role of A-Oincompatible blood transfusions in porcine orthotopic liver transplantations. Eur Surg Res 16:354-359.

Kamuf J, Garcia-Bardon A, Duenges B, Liu T, Jahn-Eimermacher A, Heid F, David M, and Hartmann EK. 2017. Endexpiratory lung volume measurement correlates with the ventilation/perfusion mismatch in lung injured pigs. Respir Res 18:101. 10.1186/s12931-017-0585-y

Kamuf J, Garcia-Bardon A, Ziebart A, Thomas R, Folkert K, Frauenknecht K, Thal SC, and Hartmann EK. 2018. Lung injury does not aggravate mechanical ventilation-induced early cerebral inflammation or apoptosis in an animal model. PLoS One 13:e0202131. 10.1371/journal.pone.0202131

Klein KU, Boehme S, Hartmann EK, Szczyrba M, Heylen L, Liu T, David M, Werner C, Markstaller K, and Engelhard K. 2013. Transmission of arterial oxygen partial pressure oscillations to the cerebral microcirculation in a porcine model of acute lung injury caused by cyclic recruitment and derecruitment. Br J Anaesth 110:266-273. 10.1093/bja/aes376

Klein KU, Johannes A, Bruckner M, Thomas R, Matthews S, Frauenknecht K, Leukel P, Mazur J, Poplawski A, Muellenbach R, Sommer CJ, Thal SC, and Engelhard K. 2016. Systemic PaO2 Oscillations Cause Mild Brain Injury in a Pig Model. Crit Care Med 44:e253-263. 10.1097/ccm.0000000000001399

Lyhs L, and Wachtel W. 1966. [On the effect of physical stress on various circulatory functions in the pig]. Arch Exp Veterinarmed 20:1057-1063.

Mascia L, Zavala E, Bosma K, Pasero D, Decaroli D, Andrews P, Isnardi D, Davi A, Arguis MJ, Berardino M, and Ducati A. 2007. High tidal volume is associated with the development of acute lung injury after severe brain injury: an international observational study. Crit Care Med 35:1815-1820. 10.1097/01.Ccm.0000275269.77467.Df

Masuda R, lijima T, Kondo R, Itoda Y, Matsuhashi M, Hashimoto S, Kohira T, Kobayashi N, and Okazaki H. 2018. Preceding haemorrhagic shock as a detrimental risk factor for respiratory distress after excessive allogeneic blood transfusion. Vox Sang 113:51-59. 10.1111/vox.12560

Mrozek S, Constantin JM, and Geeraerts T. 2015. Brain-lung crosstalk: Implications for neurocritical care patients. World J Crit Care Med 4:163-178. 10.5492/wjccm.v4.i3.163

Omi T, Vogeli P, Hagger C, Schelling C, Spilar S, Kajii E, Stranzinger G, and Neuenschwander S. 2003. cDNA cloning, mapping and polymorphism of the porcine Rhesus (RH) gene. Anim Genet 34:176182.

Palfi M, Berg S, Ernerudh J, and Berlin G. 2001. A randomized controlled trial oftransfusion-related acute lung injury: is plasma from multiparous blood donors dangerous? Transfusion 41:317-322.

Patel NN, Lin H, Toth T, Welsh GI, Jones C, Ray P, Satchell SC, Sleeman P, Angelini GD, and Murphy GJ. 2011. Reversal of anemia with allogenic RBC transfusion prevents post-cardiopulmonary bypass 
acute kidney injury in swine. American Journal of Physiology: Renal Physiology 301:F605-614. 10.1152/ajprenal.00145.2011

Pelosi P, and Rocco PR. 2011. The lung and the brain: a dangerous cross-talk. Crit Care 15:168. $10.1186 /$ cc10259

Quilez ME, Fuster G, Villar J, Flores C, Marti-Sistac O, Blanch L, and Lopez-Aguilar J. 2011. Injurious mechanical ventilation affects neuronal activation in ventilated rats. Crit Care 15:R124. $10.1186 /$ cc10230

Roubinian NH, Hendrickson JE, Triulzi DJ, Gottschall JL, Chowdhury D, Kor DJ, Looney MR, Matthay MA, Kleinman SH, Brambilla D, and Murphy EL. 2017. Incidence and clinical characteristics of transfusion-associated circulatory overload using an active surveillance algorithm. Vox Sang 112:56-63. 10.1111/vox.12466

Sachs UJ, Hattar K, Weissmann N, Bohle RM, Weiss T, Sibelius U, and Bux J. 2006. Antibody-induced neutrophil activation as a trigger for transfusion-related acute lung injury in an ex vivo rat lung model. Blood 107:1217-1219. 10.1182/blood-2005-04-1744

SchmickI CN, Mastrobuoni S, Filippidis FT, Shah S, Radic J, Murad MH, Toy P, and Gajic O. 2015. Malepredominant plasma transfusion strategy for preventing transfusion-related acute lung injury: a systematic review. Crit Care Med 43:205-225. 10.1097/ccm.0000000000000675

Sheil AG, Halliday JP, Drummond JM, Bookallil MJ, Gaudry PL, and Yezerski SD. 1972. A modified technique for orthotopic liver transplantation. Arch Surg 104:720-724.

Smith DM, Newhouse M, Naziruddin B, and Kresie L. 2006. Blood groups and transfusions in pigs. Xenotransplantation 13:186-194. 10.1111/j.1399-3089.2006.00299.x

Solomon SB, Wang D, Sun J, Kanias T, Feng J, Helms CC, Solomon MA, Alimchandani M, Quezado M, Gladwin MT, Kim-Shapiro DB, Klein HG, and Natanson C. 2013. Mortality increases after massive exchange transfusion with older stored blood in canines with experimental pneumonia. Blood 121:1663-1672. 10.1182/blood-2012-10-462945

Toy P, Popovsky MA, Abraham E, Ambruso DR, Holness LG, Kopko PM, McFarland JG, Nathens AB, Silliman CC, and Stroncek D. 2005. Transfusion-related acute lung injury: definition and review. Crit Care Med 33:721-726.

Wolsk E, Bakkestrom R, Kristensen CB, Aagaard Myhr K, Thomsen JH, Balling L, Andersen MJ, Dahl JS, Shah SJ, Gustafsson F, Hassager C, and Moller JE. 2019. Right Ventricular and Pulmonary Vascular Function are Influenced by Age and Volume Expansion in Healthy Humans. J Card Fail 25:51-59. 10.1016/j.cardfail.2018.11.013

Wozniak MJ, Qureshi S, Sullo N, Dott W, Cardigan R, Wiltshire M, Nath M, Patel NN, Kumar T, Goodall AH, and Murphy GJ. 2018. A Comparison of Red Cell Rejuvenation versus Mechanical Washing for the Prevention of Transfusion-associated Organ Injury in Swine. Anesthesiology 128:375-385. 10.1097/aln.0000000000001973

Yilmaz S, Yildirim Y, Taylan M, Demir M, Yilmaz Z, Kara AV, Aydin F, Sen HS, Karabulut A, and Topcu F. 2016. The Relationship of Fluid Overload as Assessed by Bioelectrical Impedance Analysis with Pulmonary Arterial Hypertension in Hemodialysis Patients. Med Sci Monit 22:488-494. $10.12659 / \mathrm{msm} .896305$

Ziebart A, Garcia-Bardon A, Kamuf J, Thomas R, Liu T, Schad A, Duenges B, David M, and Hartmann EK. 2015. Pulmonary effects of expiratory-assisted small-lumen ventilation during upper airway obstruction in pigs. Anaesthesia 70:1171-1179. 10.1111/anae.13154

Ziebart A, Hartmann EK, Thomas R, Liu T, Duenges B, Schad A, Bodenstein M, Thal SC, and David M. 2014. Low tidal volume pressure support versus controlled ventilation in early experimental sepsis in pigs. Respir Res 15:101. 10.1186/s12931-014-0101-6

Ziebart A, Möllmann C, Garcia-Bardon A, Kamuf J, Schäfer M, Thomas R, and Hartmann EK. 2018. Effect of gelatin-polysuccinat on cerebral oxygenation and microcirculation in a porcine haemorrhagic 
Figure 1

Figure 1: Experimental flow chart

After preparation and baseline measurement the animals were observed for eight hours. The hemodynamic and spirometric parameters were recorded continuously. Blood samples were taken every two hours. EIT (electrical impedance tomography) was measured hourly. Histopathological analysis of the lung was performed after the end of the experiment. Pulmonary and cerebral mRNA expression was quantified. (Transfusion $n=7$; Control $n=4$ )
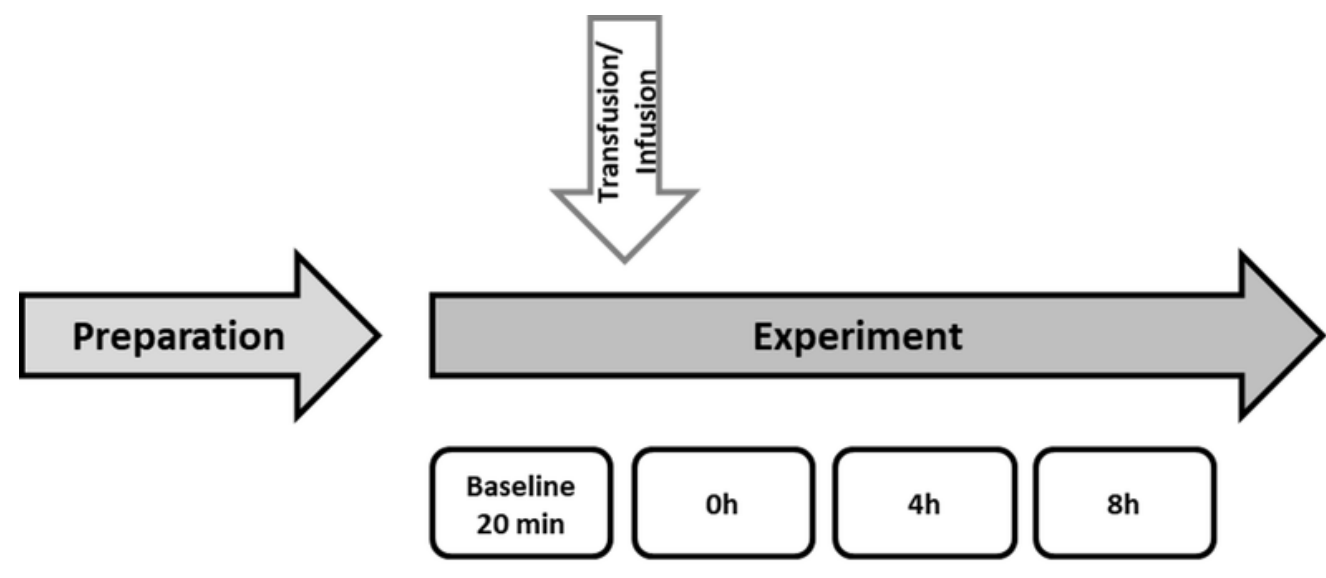

Post mortem

EIT

- Blood samples

- Vascular access

- Hemodynamic/spirometric monitoring

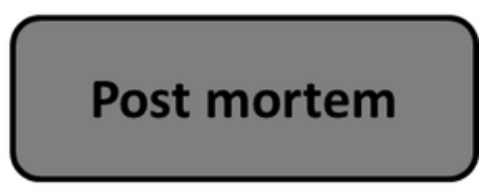

- Histopathology

- Molecular Biology

- Blood samples 


\section{Figure 2}

Figure 2: Hematological parameters.

Parameters are displayed as percentage of baseline. A significant increase of hemoglobin, hematocrit and erythrocytes was observed after transfusion. The decrease of these parameters in the control group was not significant; $*=p<0.05$; vs. baseline. (Transfusion $n=7$; Control $n=4$ ) 

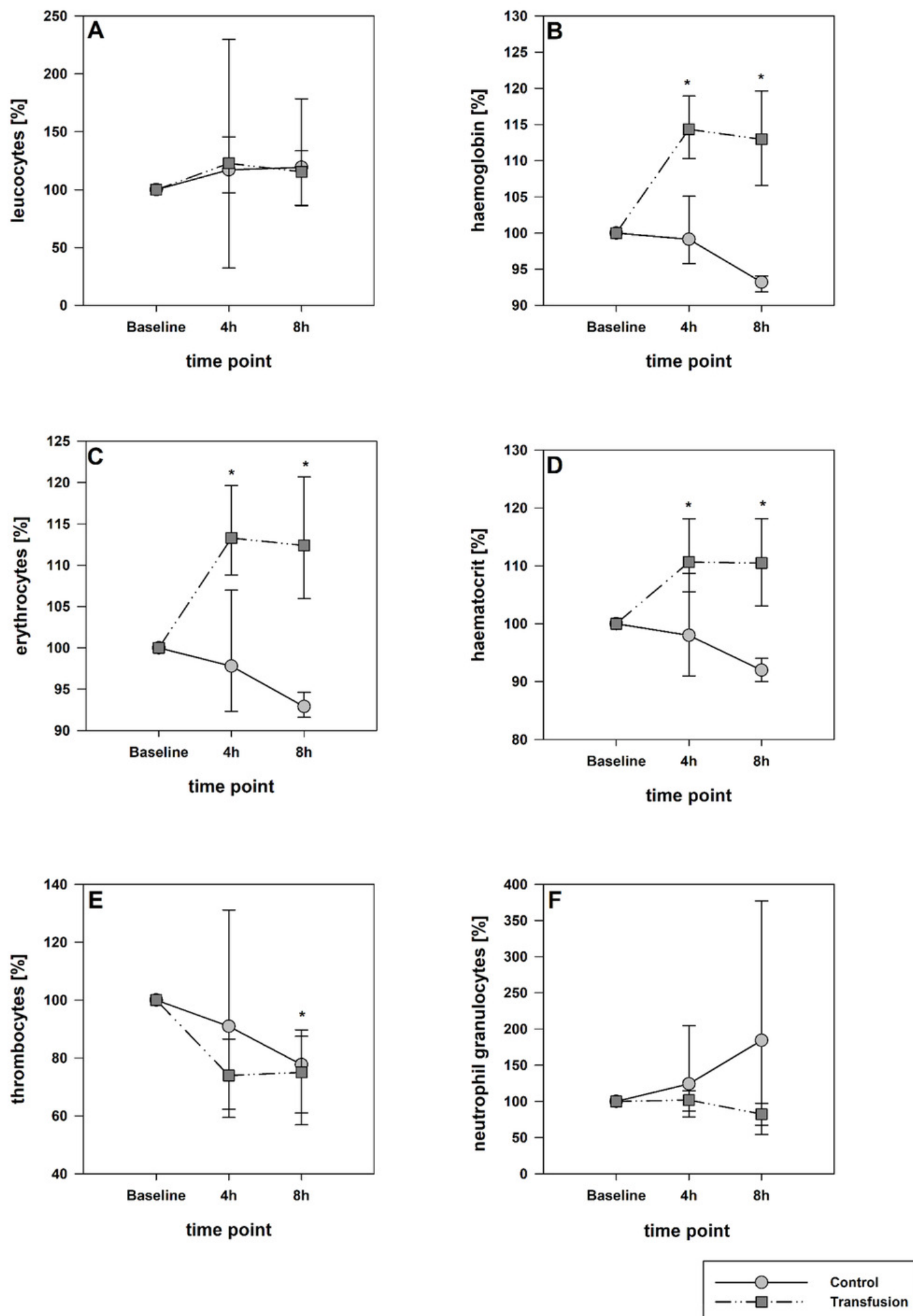
Figure 3

Figure 3: Systemic inflammatory cytokines levels.

Parameters are displayed are presented as $\mathrm{pg} / \mathrm{mL}$. The systemic inflammatory cytokines level did not differ between both study groups, but there is a significant decrease of TNFalpha to baseline in both groups; $*=p<0.05$; vs. baseline. (Transfusion $n=7$; Control $n=4$ )
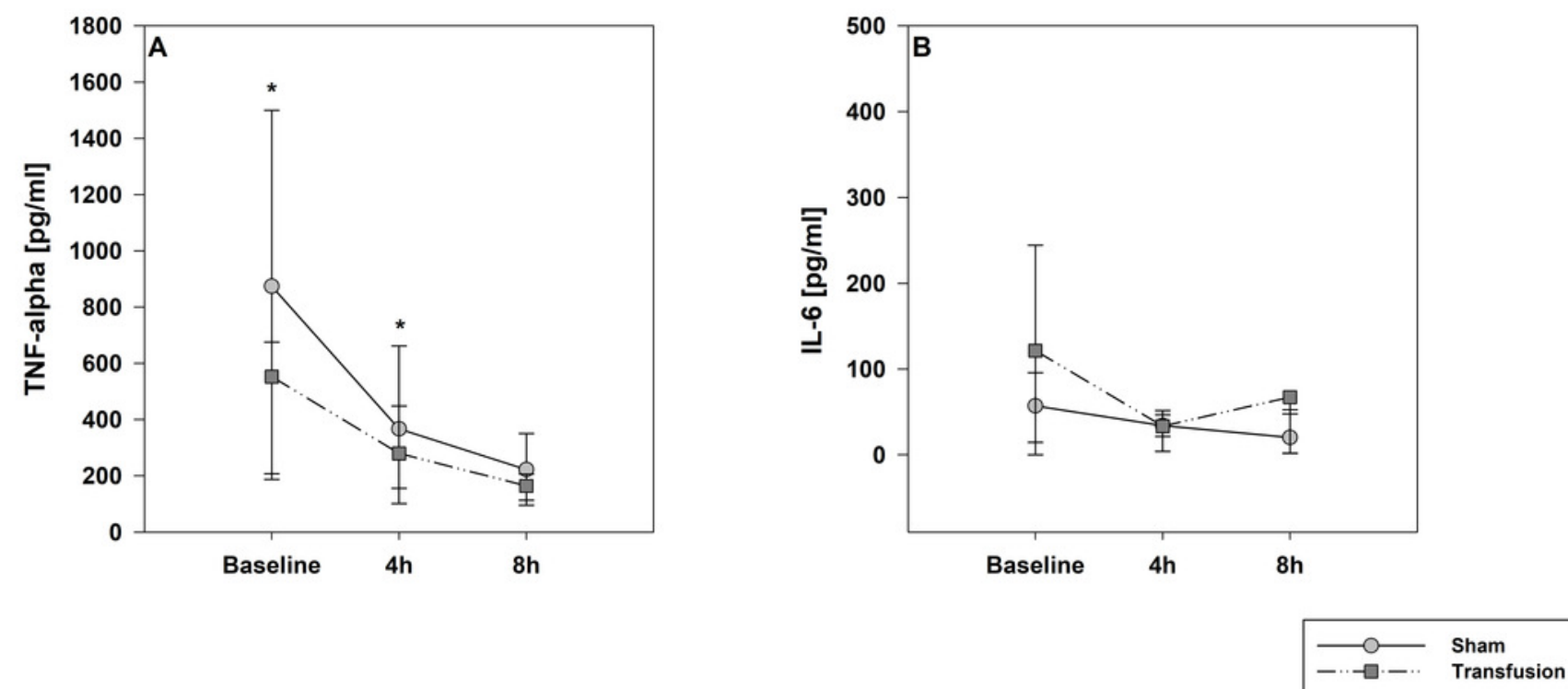
Figure 4

Figure 4: Regional distribution of the tidal volume.

Non-dependent, central and dependent lung areas (\% of the global tidal amplitude). No significant differences (each $p>0.05$ ). The regional distribution of the tidal volume did not differ between both study groups. (Transfusion $n=7$; Control $n=4$ )
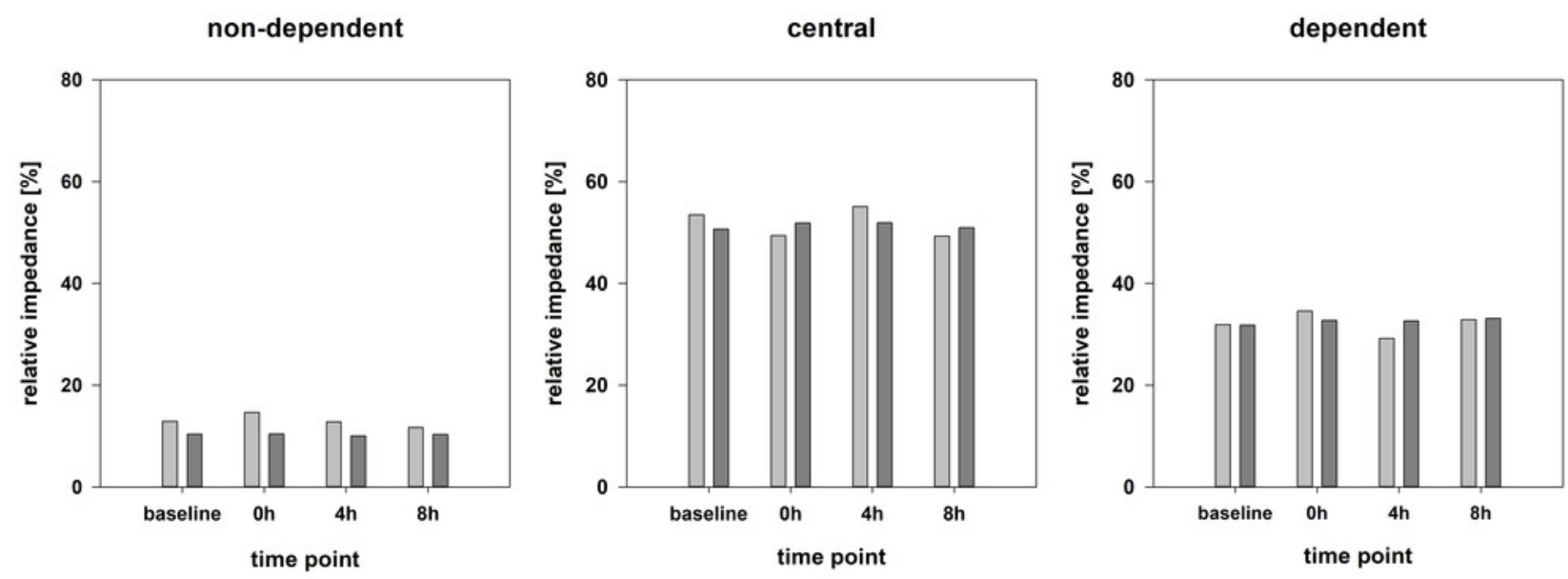


\section{Figure 5}

Figure 5: Pulmonary mRNA expression of inflammatory mediators.

Cryopreserved lung samples were collected after the experiment from upper and lower lung sections. mRNA expression of inflammatory parameters (IL-6, TNF-alpha, INOS) were analyzed via real-time polymerase chain reaction. mRNA expression is normalized to peptidylprolyl isomerase A (PPIA); no significant intergroups differences (each $p>0.05$ ). (Transfusion $\mathrm{n}=7$; Control $\mathrm{n}=4$ ). 
lower Lung
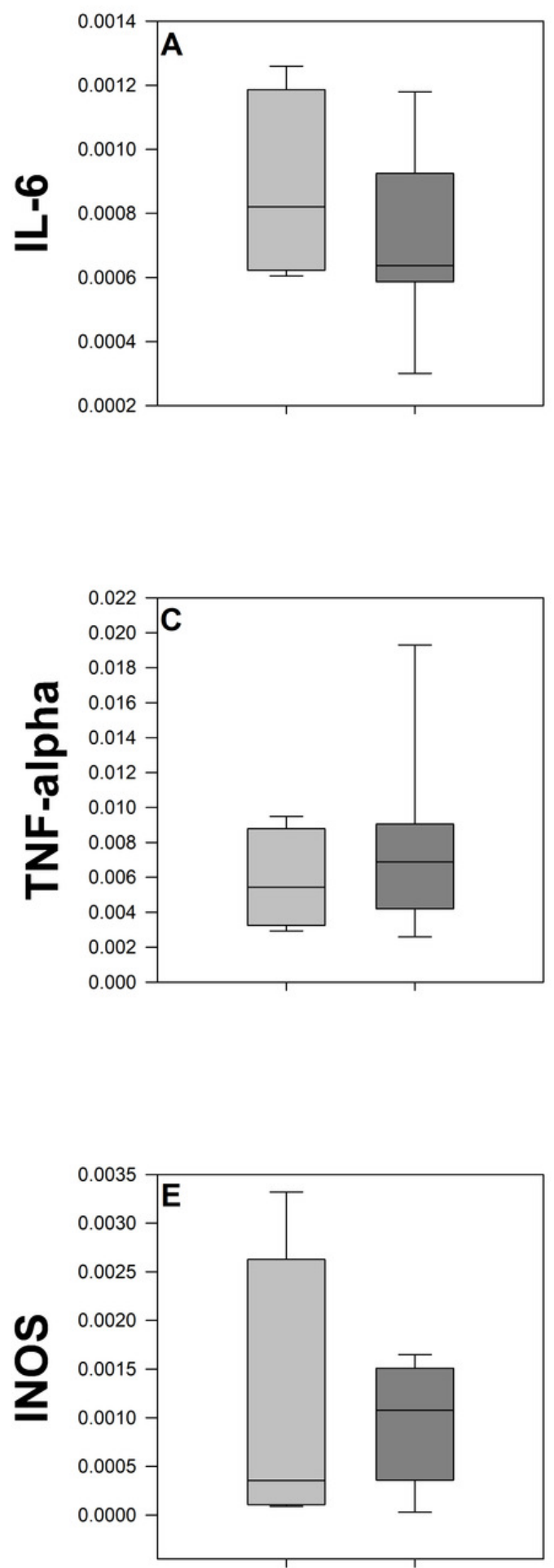

upper Lung
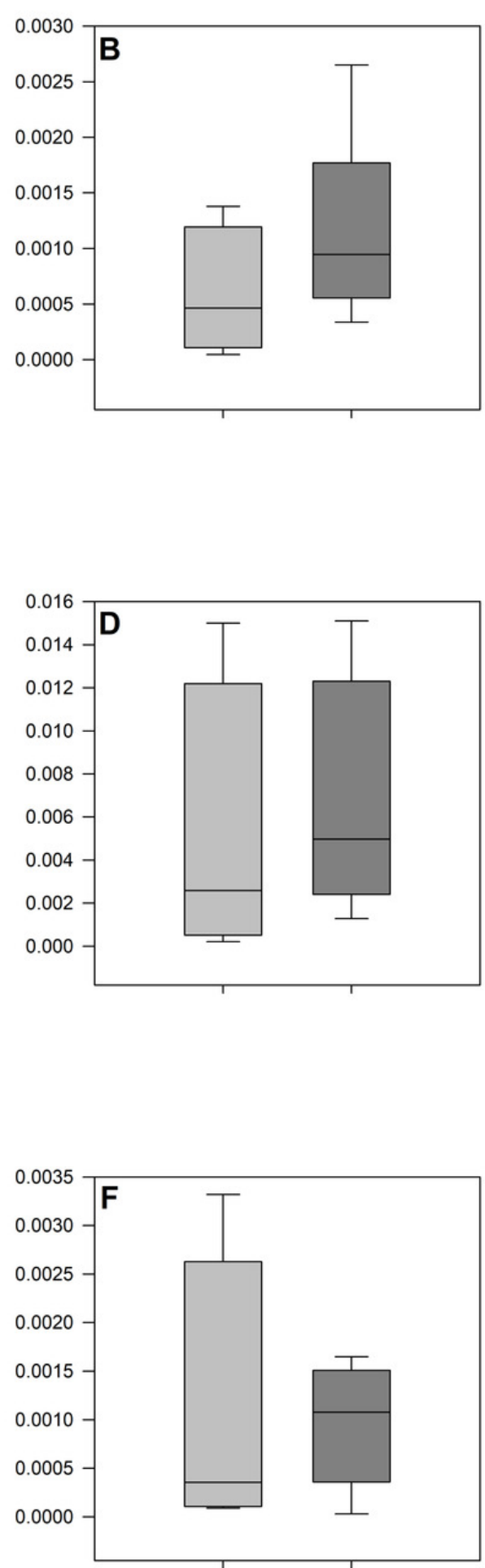


\section{Figure 6}

Figure 6: Cerebral mRNA expression of inflammatory mediators.

Cryopreserved cerebral samples were collected after the experiment from cortex and hippocampus regions. mRNA expression of inflammatory parameters (IL-6, TNF-alpha, INOS) were analyzed via real-time polymerase chain reaction. mRNA expression is normalized to peptidylprolyl isomerase A (PPIA); No significant intergroups differences (each $p>0.05$ ). (Transfusion $\mathrm{n}=7$; Control $\mathrm{n}=4$ ). 


\section{Cortex}
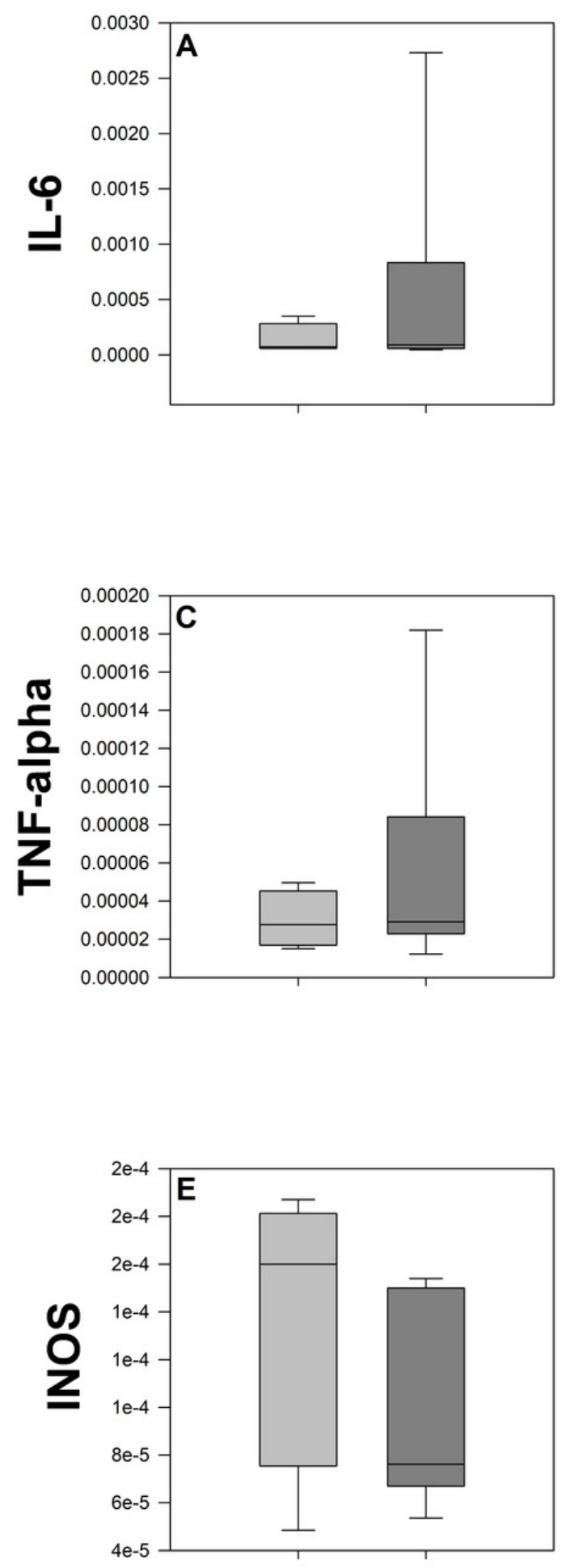

Hippocampus
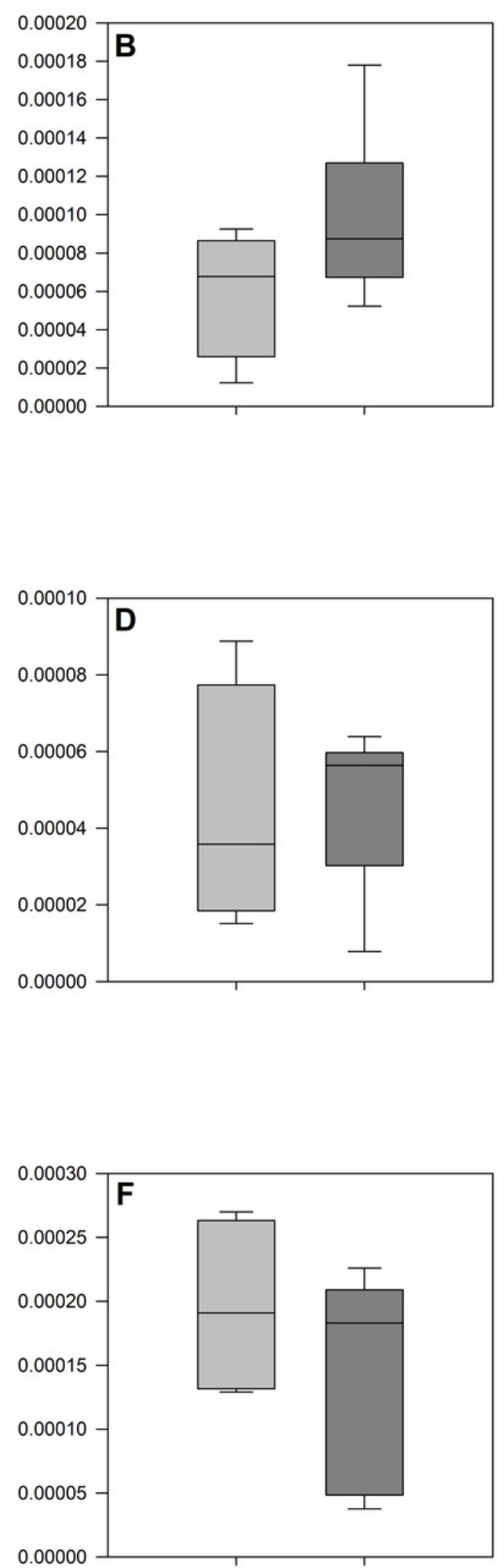


\section{Table $\mathbf{1}$ (on next page)}

Table 1: Hemodynamic parameters

No significant intra- and intergroup changes of hemodynamic parameters were observed after blood transfusion or fluid infusion, with the exception of a temporary pulmonary arterial pressure increase and raise of the central venous and mean arterial pressure after transfusion / fluid administartion. (Transfusion $n=7$; Control $n=4$ ) 


\begin{tabular}{|c|c|c|c|c|c|}
\hline Parameter & & $\begin{array}{c}\text { BLH } \\
\text { MEAN (SD) }\end{array}$ & $\begin{array}{c}\text { Oh } \\
\text { MEAN (SD) }\end{array}$ & $\begin{array}{c}\mathbf{4 h} \\
\text { MEAN (SD) }\end{array}$ & $\begin{array}{c}\mathbf{8 h} \\
\text { MEAN (SD) }\end{array}$ \\
\hline MAP & Transfusion & $70(13)$ & $98 *(14)$ & $82(9)$ & $81(14)$ \\
\hline [mmHg] & Control & $77(12)$ & $93 *(3)$ & $73(7)$ & $71(5)$ \\
\hline $\mathrm{HR}$ & Transfusion & 83 (19) & $83(13)$ & $84(20)$ & $85(13)$ \\
\hline$\left[\mathrm{min}^{-1}\right]$ & Control & $78(9)$ & $78(10)$ & $80(7)$ & $73(6)$ \\
\hline PAP & Transfusion & $18(5)$ & $27(6)^{*} \#$ & $20(2)$ & $19(3)$ \\
\hline$[\mathrm{mmHg}]$ & Control & $16(4)$ & $19(3)$ & $17(3)$ & $12(7)$ \\
\hline $\mathrm{P}_{\mathrm{es}}$ & Transfusion & $5(1)$ & $7(2)$ & $4(1)$ & $3(2)$ \\
\hline$[\mathrm{mmHg}]$ & Control & $6(0)$ & $6(1)$ & $4(1)$ & $4(0)$ \\
\hline $\mathrm{CO}$ & Transfusion & $3.6(0.7)$ & $3.7(0.7)$ & $3.2(0.3)$ & $3.2(0.6)$ \\
\hline [I $\left.\min ^{-1}\right]$ & Control & $3.4(0.3)$ & $4.3(0.9)$ & $3.4(0.3)$ & $2.9(0.4)$ \\
\hline GEDI & Transfusion & $820(125)$ & $850(96)$ & 778 (107) & 812 (81) \\
\hline$\left[\mathrm{ml} \mathrm{kg}^{-1}\right]$ & Control & 820 (97) & $916(20)$ & $808(62)$ & $803(75)$ \\
\hline EVLWI & Transfusion & $11(2)$ & $12(2)$ & $12(2)$ & $12(3)$ \\
\hline$\left[\mathrm{ml} \mathrm{kg}^{-1}\right]$ & Control & $13(5)$ & $11(0)$ & $15(3)$ & $13(2)$ \\
\hline CVP & Transfusion & $5(2)$ & $9(2)^{*}$ & $7(2)^{*}$ & $7(2)^{*}$ \\
\hline [mmHg] & Control & $7(1)$ & $8(2)$ & $7(1)$ & $7(1)$ \\
\hline $\mathrm{S}_{\mathrm{p}} \mathrm{O}_{2}$ & Transfusion & $100(0)$ & $100(0)$ & $99(1)$ & $99(1)$ \\
\hline [\%] & Control & $100(0)$ & $100(0)$ & $100(0)$ & $100(0)$ \\
\hline
\end{tabular}

*indicates $p<0.05$ vs. Baseline value. \# indicates $p<0.05$ in intergroup comparison

MAP: mean arterial pressure; HR: heart rate;

PAP: mean arterial pulmonary pressure; $\mathrm{P}_{\mathrm{es}}$ : esophageal pressure;

CO: cardiac output; GEDI: global enddiastolic volume index;

EVLWI: extravascular lung water index; CVP: central venous pressure;

$\mathrm{S}_{\mathrm{p}} \mathrm{O}_{2}$ : oxygen saturation 


\section{Table 2 (on next page)}

Table 2: Spirometric parameters

No significant intra- and intergroup changes of spirometric parameters were observed after blood transfusion or fluid infusion. (Transfusion $n=7$; Control $n=4$ ) 


\begin{tabular}{|c|c|c|c|c|c|}
\hline Parameter & & $\begin{array}{c}\text { BLH } \\
\text { MEAN (SD) }\end{array}$ & $\begin{array}{c}\text { Oh } \\
\text { MEAN (SD) }\end{array}$ & $\begin{array}{c}\mathbf{4 h} \\
\text { MEAN (SD) }\end{array}$ & $\begin{array}{c}\mathbf{8 h} \\
\text { MEAN (SD) }\end{array}$ \\
\hline $\mathrm{F}_{\mathrm{i}} \mathrm{O}_{2}$ & Transfusion & $39(0)$ & $39(0)$ & $39(0)$ & $39(0)$ \\
\hline [\%] & Control & $39(0)$ & $39(0)$ & $39(0)$ & $39(0)$ \\
\hline MV & Transfusion & $6.2(0.7)$ & $6.1(0.8)$ & $6.2(1)$ & $6.6(1.3)$ \\
\hline$[\mathrm{l} / \mathrm{min}]$ & Control & $6.4(1.7)$ & $5.8(1.3)$ & 6.5 (1.6) & $7(1)$ \\
\hline TV & Transfusion & $8(0)$ & $8(0)$ & $8(0)$ & $8(0)$ \\
\hline$\left[\mathrm{ml} \mathrm{kg}^{-1}\right]$ & Control & $8(1)$ & $8(1)$ & $8(1)$ & $8(1)$ \\
\hline$P_{\text {peak }}$ & Transfusion & $17(2)$ & $18(2)$ & $20(2)$ & $20(2)$ \\
\hline$\left[\mathrm{cm} \mathrm{H} \mathrm{H}_{2} \mathrm{O}\right]$ & Control & $16(2)$ & $17(1)$ & $17(3)$ & $19(3)$ \\
\hline $\mathrm{P}_{\text {mean }}$ & Transfusion & $9(1)$ & $9(0)$ & $10(1)$ & $10(1)$ \\
\hline$\left[\mathrm{cm} \mathrm{H} \mathrm{H}_{2} \mathrm{O}\right]$ & Control & $9(1)$ & $8(0)$ & $9(1)$ & $10(1)$ \\
\hline PEEP & Transfusion & $4(0)$ & $4(0)$ & $4(0)$ & $4(0)$ \\
\hline$\left[\mathrm{cm} \mathrm{H} \mathrm{H}_{2} \mathrm{O}\right]$ & Control & $4(0)$ & $4(0)$ & $4(0)$ & $4(0)$ \\
\hline$C_{p}$ & Transfusion & $19(3)$ & $17(2)$ & $16(2)$ & $16(3)$ \\
\hline$\left[\mathrm{ml} / \mathrm{cmH}_{2} \mathrm{O}\right]$ & Control & $20(3)$ & $20(2)$ & $19(3)$ & $17(3)$ \\
\hline $\mathrm{R}_{\mathrm{AW}}$ & Transfusion & $10(1)$ & $11(1)$ & $12(1)$ & $12(1)$ \\
\hline$[\mathrm{kPA} / \mathrm{l} / \mathrm{sec}]$ & Control & $11(1)$ & $11(1)$ & $11(1)$ & $11(2)$ \\
\hline FRC & Transfusion & $728(206)$ & $691(260)$ & $691(240)$ & 714 (235) \\
\hline$[\mathrm{ml}]$ & Control & 823 (369) & $610(87)$ & $674(77)$ & 788 (351) \\
\hline
\end{tabular}

${ }^{*}$ indicates $p<0.05$ vs. Baseline value. \# indicates $p<0.05$ in intergroup comparison

$\mathrm{F}_{\mathrm{i}} \mathrm{O}_{2}$ : fraction of inspired oxygen; $\mathrm{MV}$ : minute volume; TV: tidal volume;

Ppeak: peak inspiratory pressure; Pmean: mean airway pressure; PEEP: positive end-expiratory pressure $C_{p}$ : pulmonary compliance;

$\mathrm{R}_{\mathrm{AW}}$ : airway resistance; FRC: functional residual capacity 
1

Peer) reviewing PDF | (2018:10:31883:2:0:NEW 23 Jun 2019) 


\section{Table 3 (on next page)}

Table 3: Blood gas parameter

No relevant intra- and intergroup changes of blood gas parameters were observed after blood transfusion or fluid infusion. Only the lactate levels decrease within the normsl range and the $\mathrm{AaDO}_{2}$ increases in both groups. (Transfusion $\mathrm{n}=7$; Control $\mathrm{n}=4$ ) 


\begin{tabular}{|c|c|c|c|c|c|}
\hline Parameter & & $\begin{array}{c}\text { BLH } \\
\text { MEAN (SD) }\end{array}$ & $\begin{array}{c}\text { Oh } \\
\text { MEAN (SD) }\end{array}$ & $\begin{array}{c}\mathbf{4 h} \\
\text { MEAN (SD) }\end{array}$ & $\begin{array}{c}\mathbf{8 h} \\
\operatorname{MEAN}(S D)\end{array}$ \\
\hline $\mathrm{S}_{\mathrm{v}} \mathrm{O}_{2}$ & Transfusion & $60(12)$ & $66(8)$ & $58(6)$ & $56(6)$ \\
\hline [\%] & Control & $63(13)$ & $64(0)$ & $63(11)$ & $53(8)$ \\
\hline $\mathrm{pH}$ & Transfusion & $\begin{array}{c}7.47 \\
(0.028)\end{array}$ & $\begin{array}{c}7.505 \\
(0.027)\end{array}$ & $\begin{array}{c}7.503 \\
(0.017)\end{array}$ & $\begin{array}{c}7.495 \\
(0.019)\end{array}$ \\
\hline & Control & $\begin{array}{l}7.485 \\
(0.02)\end{array}$ & $7.5(0.033)$ & $\begin{array}{c}7.545 \\
(0.054)\end{array}$ & $\begin{array}{c}7.506 \\
(0.069)\end{array}$ \\
\hline BE & Transfusion & $4.5(4)$ & $7.3(1.4)$ & $8.5(2.2)$ & $6.6(3.6)$ \\
\hline$[\mathrm{mmol} / \mathrm{ml}]$ & Control & $5.9(4.5)$ & $6.1(0)$ & $8.9(2.4)$ & $6.4(3.4)$ \\
\hline${ }_{\mathrm{art}} \mathrm{CO}_{2}$ & Transfusion & $40(4)$ & $40(4)$ & $41(3)$ & $40(5)$ \\
\hline [mmHg] & Control & $39(5)$ & $41(4)$ & $38(6)$ & $38(4)$ \\
\hline${ }_{\mathrm{et}} \mathrm{CO}_{2}$ & Transfusion & 37 (3) & $38(3)$ & $40(1)$ & $39(3)$ \\
\hline$[\mathrm{mmHg}]$ & Control & $35(5)$ & $35(4)$ & $34(5)$ & $35(4)$ \\
\hline $\mathrm{avCO}_{2}$ diff & Transfusion & $9(3)$ & $8(4)$ & $8(2)$ & $5(6)$ \\
\hline [mmHg] & Control & $10(5)$ & $-8(21)$ & $7(1)$ & $8(1)$ \\
\hline $\mathrm{PaO}_{2}$ & Transfusion & $185(34)$ & $181(23)$ & $154(33)$ & $141(26)$ \\
\hline$[\mathrm{mmHg}]$ & Control & $206(43)$ & 230 (49) & $186(44)$ & $171(24)$ \\
\hline $\mathrm{PaO}_{2} / \mathrm{F}_{i} \mathrm{O}_{2}$ & Transfusion & $464(86)$ & $452(58)$ & $386(81)$ & $352(64)$ \\
\hline$[\mathrm{mmHg}]$ & Control & $514(108)$ & $576(122)$ & 464 (109) & $428(60)$ \\
\hline $\mathrm{AaDO}_{2}$ & Transfusion & $53(30)$ & $58(22)$ & $82(32) *$ & $97(27)^{*}$ \\
\hline [mmHg] & Control & $33(44)$ & $7(46)$ & $55(45)$ & $69(27)^{*}$ \\
\hline Temp. & Transfusion & $37.1(0.8)$ & $36.9(1.2)$ & $38(0.8)$ & $38.5(0.4)$ \\
\hline$\left[{ }^{\circ} \mathrm{C}\right]$ & Control & 35.8 (2.9) & 35.4 (3.9) & $37.6(2.2)$ & $38.3(0.6)$ \\
\hline Potassium & Transfusion & $4(0.3)$ & $3.6(0.7)$ & $4.2(0.7)$ & $3.9(0.6)$ \\
\hline$[\mathrm{mmol} / \mathrm{l}]$ & Control & $4(0.6)$ & $4(0.1)$ & $4.5(0.3)$ & $4.2(0.2)$ \\
\hline Lactate & Transfusion & $1.4(0.5)$ & $1.1(0.4)$ & $0.7(0.2)^{*}$ & $0.6(0.2)^{*}$ \\
\hline$[\mathrm{mmol} / \mathrm{l}]$ & Control & $1.4(0.4)$ & $1.3(0.1)$ & $0.9(0.2)^{*}$ & $0.8(0.3)^{*}$ \\
\hline
\end{tabular}


*indicates $p<0.05$ vs. Baseline value. \# indicates $p<0.05$ in intergroup

comparison

$\mathrm{S}_{\mathrm{v}} \mathrm{O}_{2}$ : central venous oxygen saturation, $\mathrm{BE}$ : base excess;

art. $\mathrm{CO}_{2}$ : arterial carbon dioxide; ex.CO2: exspiratory carbon dioxide; $\mathrm{avCO}_{2}$ diff: arterial venous carbon dioxide difference;

$\mathrm{PaO}_{2}$ : arterial oxygen; $\mathrm{PaO}_{2} / \mathrm{F}_{\mathrm{i}} \mathrm{O}_{2}$ : Horovitz index;

$\mathrm{AaDO}_{2}$ : alveolar-arterial oxygen difference; Temp.: Temperature 